Int. J. Electrochem. Sci., 5 (2010) 377 - 391

International Journal of

ELECTROCHEMICAL

SCIENCE

www.electrochemsci.org

\title{
Fast Fourier Transformation with Continuous Cyclic Voltammetry at Pt-Au Dual Microelectrode for the Determination of Chloramphenicol in a Flow Injection System
}

\author{
P. Norouzi, ${ }^{* 1,2}$ H. Rashedi, ${ }^{3}$ T. Mirzaei Garakani, ${ }^{1}$ R. Mirshafian ${ }^{1}$ and M.R. Ganjali ${ }^{2,1}$ \\ ${ }^{1}$ Center of Excellence in Electrochemistry, Department of Chemistry, University of Tehran, Tehran, \\ Iran \\ ${ }^{2}$ Endocrinology \& Metabolism Research Center, Tehran University of Medical Sciences, Tehran, Iran \\ ${ }^{3}$ Department of Chemical Engineering, Faculty of Engineering, University of Tehran, Tehran, Iran \\ *E-mail: norouzi@khayam.ut.ac.ir
}

Received: 3 March 2010 / Accepted: 15 March 2010 / Published: 31 March 2010

Fast Fourier transformation with continuous cyclic voltammetry (FFTCV) in a flowing solution was applied as a detection system for the prompt Chloramphenicol (CAP) monitoring. In this method, the potential waveform consisted of two potential sections for cleaning of the electrode surface, accumulating CAP and potential ramp, which was continuously applied on a Pt-Au dual disk microelectrodes. The effects of various parameters on the sensitivity of the method were investigated. When the optimum conditions were imposed, this is a scan rate value of $70 \mathrm{~V} / \mathrm{s}$, an accumulation time of $500 \mathrm{~ms}$, an accumulation potential of $-300 \mathrm{mV}$ and a $\mathrm{pH}$ value of 2 . The method proved to be linear over the concentration range of $8.0 \times 10^{-9}-2.0 \times 10^{-5} \mathrm{~mol} / \mathrm{L}(r=0.9981)$ with a detection limit and a quantitation limit of $2.0 \times 10^{-9}$ and $6.6 \times 10^{-9} \mathrm{~mol} / \mathrm{L}$, respectively. Consequently, the method illustrates the requisite accuracy, sensitivity, precision and selectivity to assay CAP in its biological fluids. The proposed method has some advantages over other reported methods such as, no need for the removal of oxygen from the test solution, a sub-nanomolar detection limit, and finally the method is fast enough for the determination of any such compound, in a wide variety of chromatographic methods. As a result, FFTCV illustrates the benefits of precision, determination speed, cost-effectiveness, accuracy and simplicity, in comparison with formerly reported techniques.

Keywords: Chloramphenicol, Fast Fourier transformation Continuous cyclic voltammetry, Flow injection, Pt-Au dual disk microelectrode

\section{INTRODUCTION}

Antibiotics like Chloramphenicol (CAP) are widely used in veterinary practice as feed additives for growth promotion because of their broad range of activity against bacteria and low cost. Its chemical structure is shown in Scheme 1. 


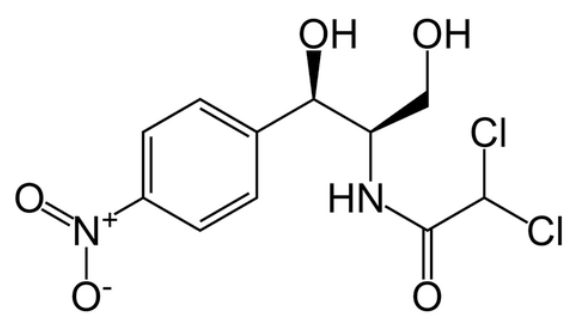

Scheme 1. Chemical structure of CAP

Adverse reactions and side effects in humans have been extensively demonstrated [1]; therefore, to protect the consumer, European Community banned use of CAP in food producing animals and established a zero tolerance level for this compound in edible tissues [2]. In order to effectively monitor the occurrence of residues of CAP, specific and sensitive analytical methods are required. For screening purposes, immunological methods are claimed to be very sensitive, but positive results need further confirmation [3]. Several techniques have been developed for determination of CAP in different biological materials. They are photoinduced chemiluminescence [4], high performance liquid chromatography (HPLC) with UV, single MS and tandem MS detection [5], capillary zone electrophoresis with amperometric detection [6], and surface plasmon resonance biosensor [7]. Although these methods have advantages of sensitivity and accuracy, their high cost and complicated operation limit their extensive application. Electrochemical methods are simple, speedy, sensitive and inexpensive, thus they are attempted for CAP determination. Agui et al. [8] developed a voltammetric method for CAP with electrochemically activated carbon fiber microelectrodes; the linear range was $1.0 \times 10^{-7}-1.0 \times 10^{-5} \mathrm{~mol} / \mathrm{L}$ and the detection limit was $4.7 \times 10^{-8} \mathrm{~mol} / \mathrm{L}$. Chen et al. [9] developed a novel one-off amperometric sensor incorporating a three-electrode configuration for direct detection of CAP, and the detection limit was $4.2 \times 10^{-7} \mathrm{~mol} / \mathrm{L}$.

In pharmaceutical, biomedical and food analysis, a tendency can be observed towards the development of miniaturized and fast methods to achieve a highly sensitive determination. Recently, stripping voltammetric methods were used in determination of heavy metal ions and some organic compounds in flowing solutions with a parts-per-billion sensitivity range. In addition, owing to the movement of the analyte zone in an electrochemical flow cell for flowing solutions, the application of these techniques requires fast analyte accumulation and fast potential sweeping (which is not appropriate for large electrodes) [10,11]. So the ultramicroelectrodes (UMEs) introduction has encouraged the use of voltammetric techniques. In addition, it should be noted that this novel electrochemical method has many advantages over the classical methods. For example, they demonstrate steady state currents, higher sensitivity caused by the increased mass transport and application to highly resistant solutions. Additionally, UMEs have been applied as sensors in various techniques such as flow injection analysis $[12,13]$, cardiovascular monitoring and organic compounds analysis [14-16]. For this work, we used the highly developed version of a classical cyclic voltammetric technique combined with fast Fourier transformation method (the computer program is more advanced in the algorithm for integration and filtering). In addition to improve sensitivity of the method we use dual Pt-Au dual UMEs as working electrode. This dual electrode can enhance 
absorption of CAP molecules. As a consequence, the noise level was less, resulting in a better S/N. Fortunately; it was feasible to determine CAP at a detection limit lower than those of other reported methods [17].

\section{EXPERIMENTAL PART}

\subsection{Flow injection setup}

In Figure 1, the electrochemical cell of the flow injection analysis is illustrated. For this analysis, an eight-roller peristaltic pump (v10.11, Zitteck Co. Iran) and a four-way injection valve (Supelco Rheodyne Model 5020) with a 50- $\mu$ L sample injection loop were used. In addition, a plastic syringe was used for the introduction of the solutions into the sample loop. The flow rate of the eluent solution during the experiments was set to $7 \mathrm{~mL} / \mathrm{min}$.

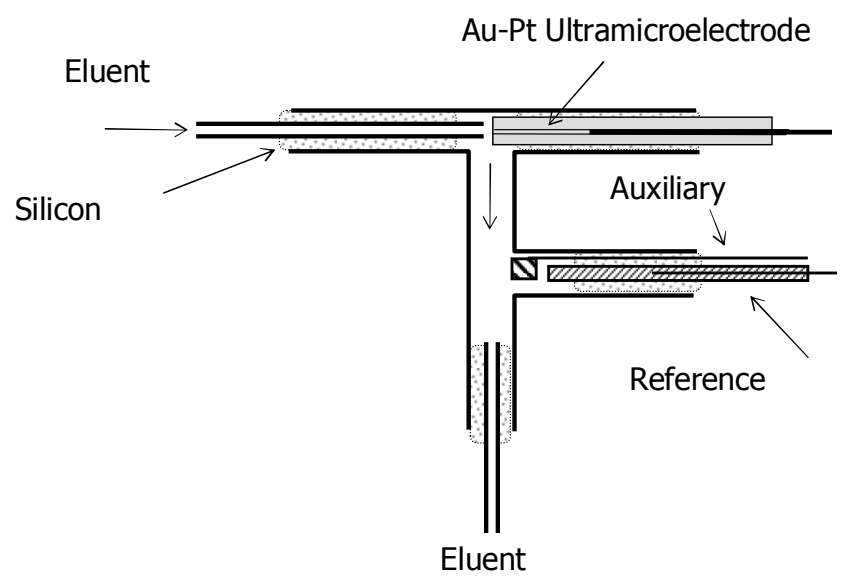

Figure 1. Diagram of the electrochemical cell

\subsection{Reagents}

For the experimental part of the research, the analytical grade reagents and the reagents for the preparation of the eluent solution for the flow injection analysis were obtained from the Merck Chemicals. The Drug was purchased from Sigma-Aldrich provided CAP. All solutions were prepared in doubly distilled deionized water, filled with the background electrolyte solution and they were used without the removal of the dissolved oxygen.

\subsection{Background electrolyte (BGE)}

The running buffer or BGE was made by phosphoric acid $(85 \% \mathrm{w} / \mathrm{v})$ with addition of $1.82 \mathrm{~mL}$ into a $1000 \mathrm{~mL}$ volumetric flask and dilution to a constant volume with distilled water. The $\mathrm{pH}$ was 
adjusted to the value of 2 by sodium hydroxide. The solutions were freshly prepared and filtered, using a Millipore filter $(0.45 \mu \mathrm{m})$ each day.

\subsubsection{Procedure for the preparation of standard}

Stock solution $(1.0 \mathrm{mg} / \mathrm{mL})$ of CAP prepared by dissolving $100 \mathrm{mg}$ of the drug sample in 100 $\mathrm{mL}$ of distilled water. This solution was further diluted daily with water to give the appropriate concentration for the working solution.

\subsubsection{Sample preparation of human urine}

Urine was collected from healthy volunteers (males, around 35 years old). An aliquot of a standard aqueous solution of CAP containing (1.0-5.0 $\mu \mathrm{g}$ ) was added to $0.5 \mathrm{~mL}$ of urine sample in a centrifuge tube and vortex for $20 \mathrm{~s}$. Fifty microliter of $0.1 \mathrm{~mol} / \mathrm{L} \mathrm{NaOH}$ solution was added, shaken for few seconds, followed by the addition of $2.5 \mathrm{~mL}$ dichloromethane. The mixture was vortex mixed at high speed for $2 \mathrm{~min}$, and then centrifuged at $3000 \mathrm{rpm}$ for $10 \mathrm{~min}$. The resulting supernatant was transferred to a small conical flask. The extract was evaporated to dryness at $60{ }^{\circ} \mathrm{C}$ and the residue was dissolved in $0.5 \mathrm{~mL}$ water, diluted with $0.05 \mathrm{~mol} / \mathrm{L}$ phosphoric acid to a $100 \mathrm{~mL}$ flask and then analyzed according to the recommended procedure. The voltammograms were recorded according to the above recommended procedure. The voltammograms of the samples without CAP did not show any signal that could interfere with the direct determination. Therefore, external calibration could be used.

\subsection{Electrode preparation}

The construction of the Pt-Au dual microelectrodes ( $25 \mu \mathrm{m}$ in radius) involved the metal microwires (Goodfellow Metals Ltd., UK) sealing into a soft glass capillary. Then, the capillary was cut perpendicularly to its length to expose the wire. Silver epoxy (Johnson Matthey Ltd., UK) was used for the electrical contacts. Previous to the initiation of any experiment, the electrode surface was polished for $1 \mathrm{~min}$ using extra fine carborundum paper and, afterwards, for $10 \mathrm{~min}$ with $0.3 \mu \mathrm{m}$ alumina. Prior to being placed in the cell, the electrode was washed with water. In all measurements, an $\mathrm{Ag}(\mathrm{s})|\mathrm{AgCl}(\mathrm{s})| \mathrm{KCl}(\mathrm{aq}, 1 \mathrm{~mol} / \mathrm{L})$ reference electrode was used. The auxiliary electrode was made of a $\mathrm{Pt}$ wire, $1 \mathrm{~cm}$ in length and $0.5 \mathrm{~mm}$ in diameter.

\subsection{Validation}

The validation parameters of the method included the selectivity, limit of quantitation (LOQ), limit of detection (LOD), robustness, accuracy precision, recovery and linearity [18-20]. Linear regression analysis was applied along with the help of the least square regression method for the evaluation of the linearity $[21,22]$. The peak areas of CAP were plotted versus its concentration and, 
afterwards, linear regression analysis was carried out on the resultant curve. The LOQ and LOD were calculated from the signal-to-noise ratios, being based on the analytical responses of the background noise for 10 and 3 times, respectively [23]. The precision assessment was performed in accordance with repeatability and reproducibility. Repeatability was investigated by injecting nine replicate samples of each of the $4 \times 10^{-8}, 9 \times 10^{-7}$ and $5 \times 10^{-6} \mathrm{~mol} / \mathrm{L}$ standards. For the accuracy assessment of the method, replicate $(n=6)$ peak areas of three accuracy standards $\left(4.6 \times 10^{-8}, 8.5 \times 10^{-7}\right.$ and $5.3 \times 10^{-6}$ $\mathrm{mol} / \mathrm{L}$ ) were interpolated from a calibration curve, which was prepared as previously reported. Additionally, the relevant error percentage and accuracy were calculated in each case.

\section{RESULTS AND DISCUSSION}

\subsection{Data acquisition and processing}

A setup of a PC PIV Pentium, equipped with a data acquisition board (PCL-818HG, Advantech. Co.) and a custom made potentiostate, was used for the appliting potential and data acquisition. All data acquisition and data processing programs were developed in Delphi 6® program environment. The applied waveform potential diagram during the voltammetric measurements is shown In Figure 2. As it is seen the potential waveform consists of three parts; (a) Potential steps, $E_{\mathrm{c} 1}$ and $E_{\mathrm{c} 2}$ (which are used for the oxidation and reduction of the electrode surface, respectively), during which the electrochemical cleaning of the electrode surface takes place, (b) $E_{\mathrm{c}}$, where the analyte accumulation takes place and (c) the potential ramp, where the current measurements occur.

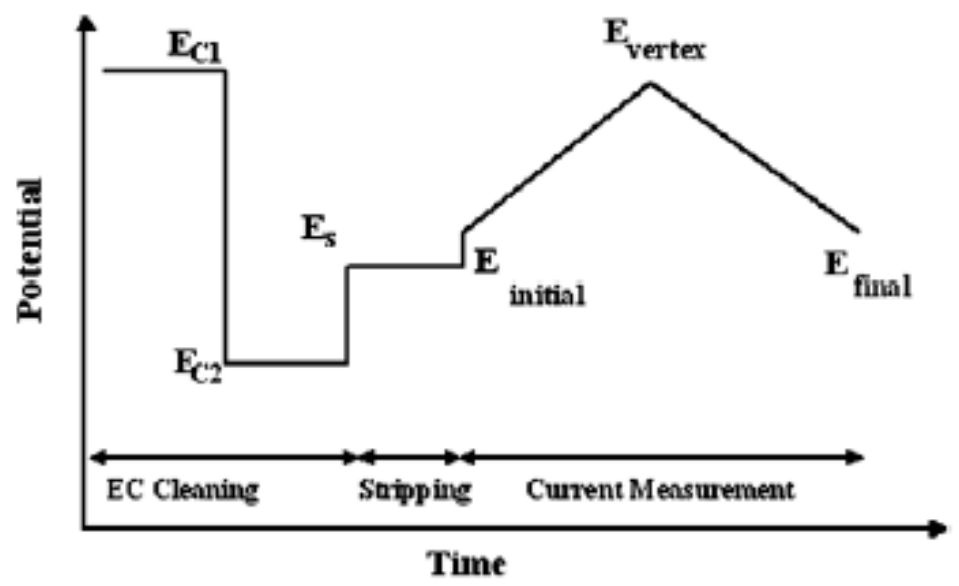

Figure 2. Applied potential waveform diagram

In this method, the signal calculation is measured by the integration of the net current changes over the scanned potential range. It should be noted that, in this case, changes in the current (result of the injected analyte) at the voltammogram can be caused by several types of processes, which could be occured on the electrode surface. Those processes include: (a) oxidation and reduction of the adsorbed analyte and (b) oxidation and reduction inhibition of the electrode surface by the adsorbed analyte. In fact, in order to observe the influence of the adsorbed analyte on the oxidation peaks of the Pt-Au 
surfaces, the sweep rate must be set at high rates (e.g. $>10 \mathrm{~V} / \mathrm{s}$ ), because in low seep rate it is possible the adsorbed analyte desorbed from the electrode surface.

The significant point here is that the adsorbed analyte molecules still remain on the electrode surface, inhibiting the oxidation-reduction process of the electrode surfaces. In this technique, $\Delta Q$ is calculated according with the all current changes at the CVs [24-28]. However, the selectivity and sensitivity of the analyte response, expressed in terms of $\Delta Q$, strongly depends on the selection of the integration limits.

One of the important outlooks of this technique is the application of a special digital filtration, applied during the measurement. Firstly, an electrode CV was recorded and then by applying FFT on the collected data, the existing high frequency noises were indicated. With the aid of this information, the cut-off frequency of the analog filter was set at a certain value (where the noises were removed from the $\mathrm{CV}$ ). Various potential waveforms were investigated in order to obtain a reproducible electrode surface (or a stable background signal) provided that the crystal structure of polycrystalline gold and platinum surfaces is greatly affected by the condition of the applied potential waveform [29]. As a result of the changes taking place in the surface crystal structure during the oxidation and reduction of the electrodes in each potential cycle, which can cause low stability of the background signal. After the application of several types designed potential waveforms, the best potential waveform, giving a stable background during application of continuous cycling, was the waveform shown in Figure 2. The starting point for the electrochemical oxidation process of the $\mathrm{Au}$ and $\mathrm{Pt}$ surfaces is the hydroxyl ion electrosorption which at more positive potentials results in the metal oxide formation, undergoing structural rearrangement [30]. In addition, oxidation of the surface can be done initially by the water molecule adsorption and, at a more positive potential, $\mathrm{AuOH}$ and $\mathrm{PtOH}$ are formed bringing to a phase formation of the metal oxide;

$$
\begin{aligned}
& 2 \mathrm{Au}+3 \mathrm{H}_{2} \mathrm{O} \rightarrow \mathrm{Au}_{2} \mathrm{O}_{3}+6 \mathrm{e}+6 \mathrm{H}^{+} \\
& \mathrm{Pt}+2 \mathrm{H}_{2} \mathrm{O} \rightarrow \mathrm{PtO}_{2}+4 \mathrm{H}^{+}+4 \mathrm{e}^{-}
\end{aligned}
$$

An example of the recorded CVs is depicted in Figure 3a and b. The previous (Figure 3a) depicts a $\mathrm{CV}$ sequence recorded during the flow analysis for the drug determination. The injection volume was $50 \mu \mathrm{L}$ of $5.0 \times 10^{-6} \mathrm{~mol} / \mathrm{L} \mathrm{CAP}$ into the eluent solution containing $0.05 \mathrm{~mol} / \mathrm{L}$ phosphoric acid. The time axis of the graph shows the time of the flow injection experiment. In solution without $\mathrm{CAP}$, the shape of the CV curves is typical for a polycrystalline Au and Pt electrode in acidic media The latter (Figure 3b) illustrates the absolute current changes in the CVs curves, after the subtraction of the four CVs average background (in the absence of the analyte). Details of adsorbed ion effect on the $\mathrm{CV}$ currents are more obvious by presenting the electrode response with this method. Actually, the curves show that the current changes mainly take place at the potential regions of the oxidation and reduction of Pt-Au. The oxide formation process becomes critically inhibited, when the electrodesolution interface is exposed to CAP, which can be adsorbed on the electrode. Then, significant change in the currents at the potential region is resulted due to the surface process inhibition and, as a consequence, the profound changes in the shape of the CVs take place. 


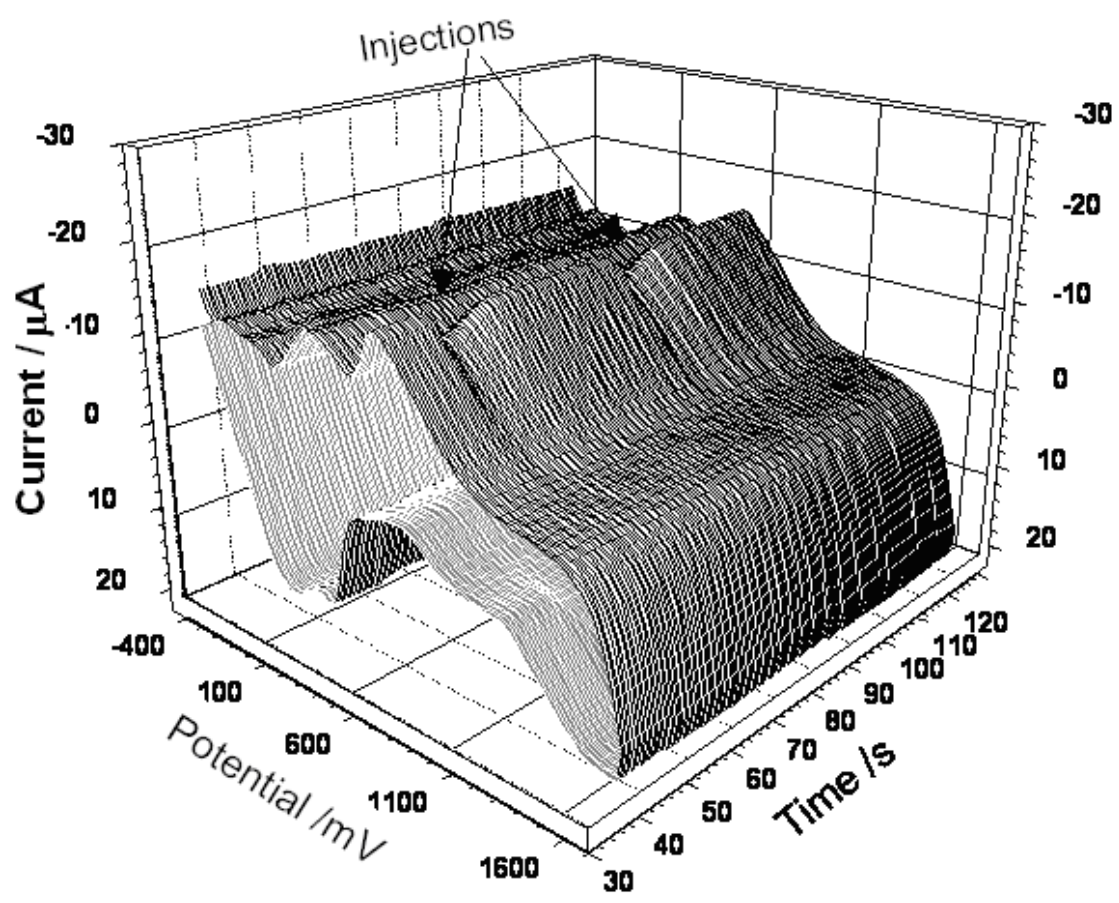

(a)

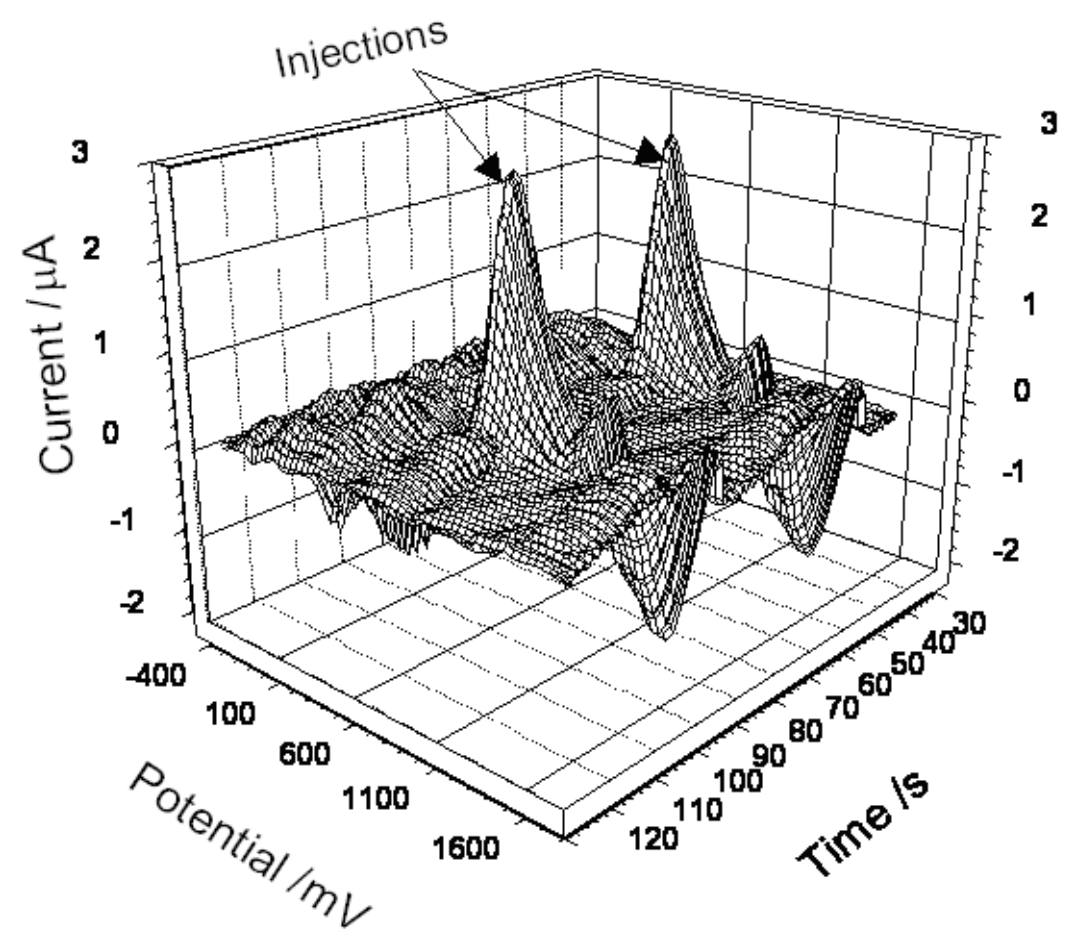

(b)

Figure 3. (a) Cyclic voltammogram at a Pt-Au UMEs, recorded during a flow-injection experiment. Each scan was preceded by a conditioning of $100 \mathrm{~ms}$ (at $1600 \mathrm{mV}$ ) and $100 \mathrm{~ms}$ (at $300 \mathrm{mV}$ ), respectively. These CVs are recorded at the optimum condition and flow rate. (b) Curves result from the subtraction of a CV average (in the absence of the analyte) from the CV test in (a) 
In chromatographic analysis, where a mixture of compounds presents in the sample, the universality of the detector is beneficial. In most cases, the analyte response can be affected by three functions including; the thermodynamic and kinetic parameters of adsorption, the mass transport rate and the electrochemical behavior of the adsorbed species. Due to that, for the achievement of the detector maximum performance, the effect of the experimental parameters (such as the $\mathrm{pH}$ of the supporting electrolyte, the potential, the accumulation time and the potential sweep rate) must be examined and optimized.

\subsection{Experimental parameter optimization}

\subsection{1. pH Effect of the eluent}

Figure 4 shows the signal of CAP on the Pt-Au UMEs in different $\mathrm{pH}$ values of the eluent. As it is shown, the $\mathrm{pH}$ of the eluent changes the potentials of oxidation and reduction of the dual UMEs. In Figure 4 it can be clearly seen that the basic solutions (high $\mathrm{pH}$ values) are not suitable for the measurements. However, the best $\mathrm{S} / \mathrm{N}$ ratio was obtained between the $\mathrm{pH}$ values of $\sim 2$. Furthermore, it is illustrated by the results that at $\mathrm{pH}$ values higher than 9 , the noise level in the baseline ( $\Delta Q$ versus Time) is higher up to $12 \%$, in comparison with that of the acidic solution.

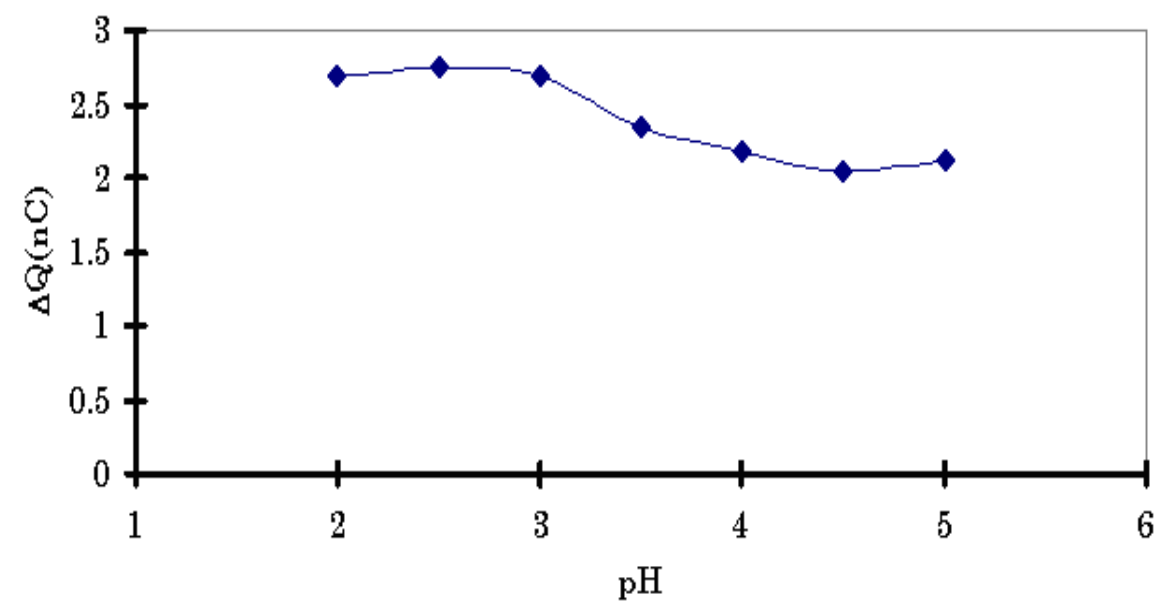

Figure 4. Cyclic voltammograms of $\mathrm{Pt}-\mathrm{Au}$ electrode in various $\mathrm{pH}$ values at the sweep rate $70 \mathrm{~V} / \mathrm{s}$

\subsubsection{Sweeping rate effect}

Nevertheless, for the investigation of the sweep rates influence and the eluent flow rate on the sensitivity of the detector response, solutions with a concentration of $\left(5.0 \times 10^{-6} \mathrm{~mol} / \mathrm{L}\right)$ in CAP were injected. Then, the detector responses to the injected sample were recorded at different sweep rates (from 10 to 90V/s) and eluent flow. These results are shown in Figure 5. In the condition that it is obvious from Figure 5, the detector shows the maximum sensitivity at the sweep rate of (70V/s) and 
flow rate of $(7 \mathrm{~mL} / \mathrm{min})$. The effects of sweep rate on the detection performance can be considered in three different points of view; the first effect affects the speed in data acquisition. The second one is the kinetic factors related to the CAP adsorption and the last one is flow rate of the eluent, which controls the time window of the injected solution zone in the detector. The most significant reason for the application of high sweep rates is inhibition of desorption of the adsorbed CAP on the electrode surfaces, while the potential is being scanned (because under this condition, the inhibition outcome of the adsorbed CAP on the oxidation/reduction peaks of Pt-Au can occur). From this point of view, it is necessary to check how the method sensitivity is affected by the sweep rate [31-43]. Furthermore, the necessity of investigating the way that the sweep rate affects the method sensitivity is indicated. Hence, high sweep rates should be applied to detect the adsorbed analyte amount on the electrode surface, in order that the potential scanning step is short when it is compared with the accumulation period.

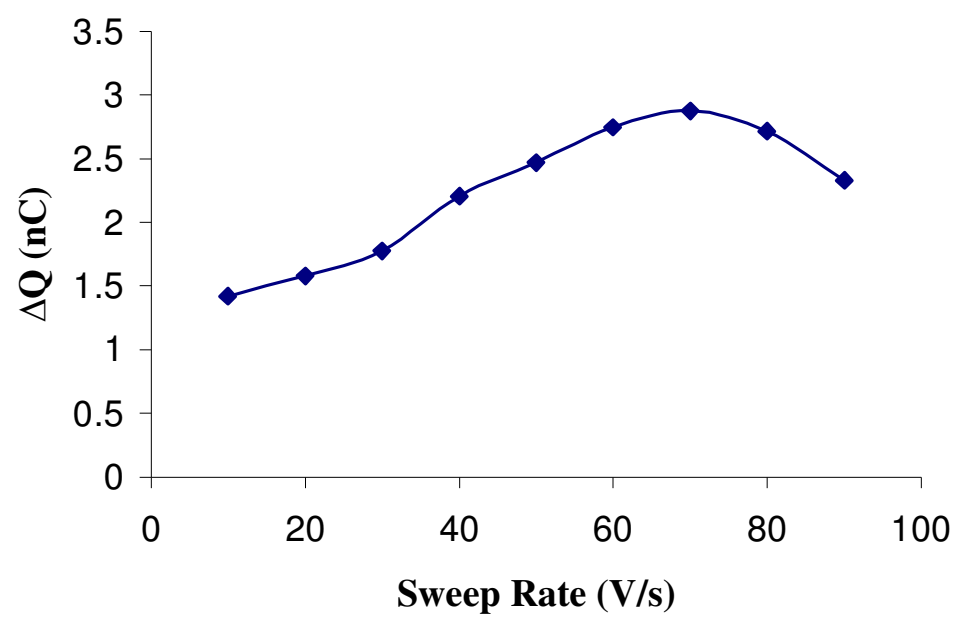

Figure 5. Sweep rate effect on the Pt-Au electrode response (with a radius of $25 \mu \mathrm{m}$ ) to the injection of $5.0 \times 10^{-6} \mathrm{~mol} / \mathrm{L}$ Chloramphenicol in $0.05 \mathrm{~mol} / \mathrm{L} \mathrm{H}_{3} \mathrm{PO}_{4}$

\subsubsection{Accumulation potential effect}

As stated before the sensitivity of the measurement, seriously is influenced by any changes in the parameters which are related to the adsorption process and affecting the applied potential, the time and the potential of accumulation. Considering that, the influence of the accumulation potential on the response of the method for the solution injection of $\left(5.0 \times 10^{-6} \mathrm{~mol} / \mathrm{L}\right) \mathrm{CAP}$, in $0.05 \mathrm{~mol} / \mathrm{L}$ phosphoric acid was studied. In Figure 6, the detector response is illustrated over the accumulation potential ranges from -500 to $200 \mathrm{mV}$. The results are depicted in Figure 6, where it is evident that the adsorption process demonstrates a high dependence upon the applied potential. It is clear that the optimum conditions to be chosen are accumulation potential of $-300 \mathrm{mV}$ on the surface of dual microelectrode. This result can be easily explained for CAP; at positive potentials, the accumulation efficiency decreases due to the cation's adsorption on the positively charged electrode becomes thermodynamically unfavorable. At negative potentials, the observed response drop can be attributed to the competitive adsorption of hydronium ions. 


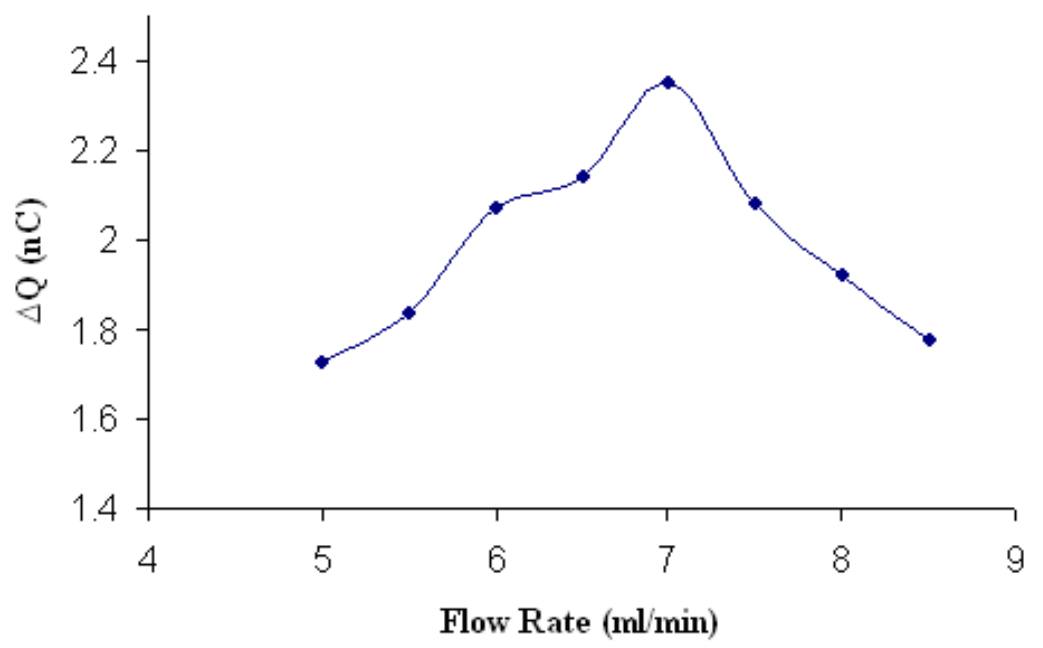

Figure 6. Accumulation potential effect on the Pt-Au electrode response to the injection of $5.0 \times 10^{-6}$ $\mathrm{mol} / \mathrm{L}$ Chloramphenicol in $0.05 \mathrm{~mol} / \mathrm{L} \mathrm{H}_{3} \mathrm{PO}_{4}$

\subsubsection{Sweeping rate effect}

The detection system sensitivity mainly depends on the accumulation time. The accumulation of CAP on the electrode surface takes place during the stripping step (assuming that an appropriate potential is selected). Figure 7 depicts the plot of peak charge versus preconcentration time for a CAP concentration of $5.0 \times 10^{-6} \mathrm{~mol} / \mathrm{L}$. As it is shown in Figure 7, the electrode surface becomes saturated within a $600-\mathrm{ms}$ time window. Considering the small size of the Pt-Au surfaces and the short time needed for saturating them, an appropriate potential is selected and the CAP accumulation takes place on the electrode during the accumulation step. In fact, the existing differences in the kinetics of the electron transfer and mass transport result in different values for the saturation time of the various compounds.

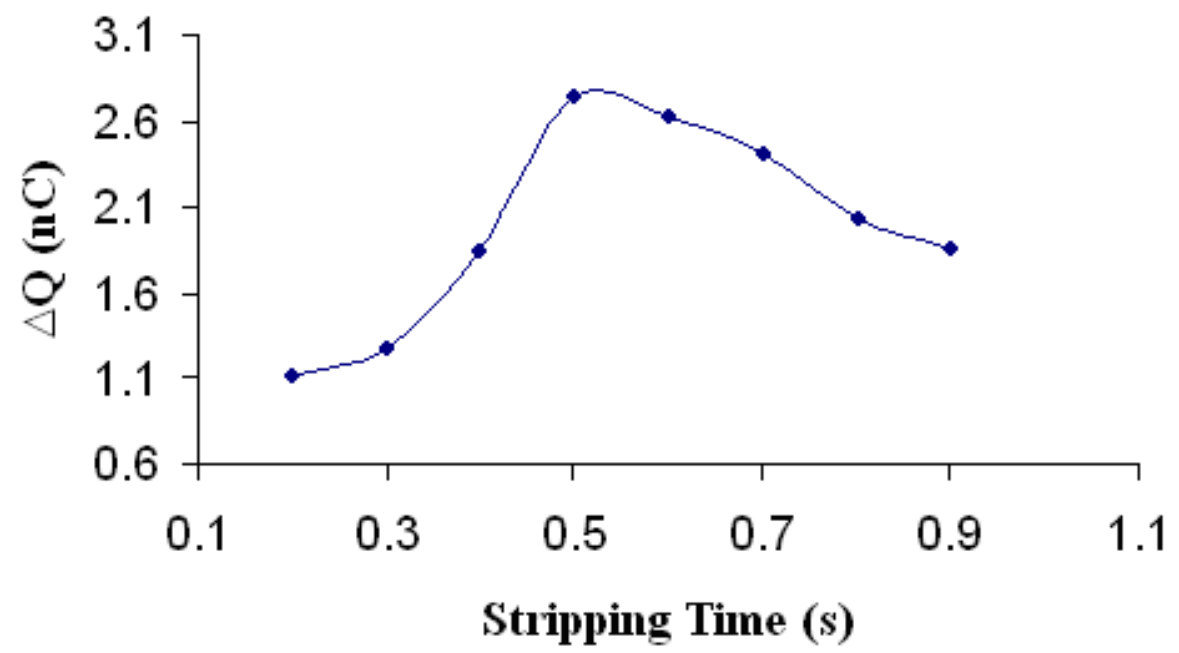

Figure 7. Accumulation time effect on the Pt-Au electrode response to the injection of $5.0 \times 10^{-6} \mathrm{~mol} / \mathrm{L}$ Chloramphenicol in $0.05 \mathrm{~mol} / \mathrm{L} \mathrm{H}_{3} \mathrm{PO}_{4}$ 


\subsection{Sweeping rate effect}

The Pt-Au UMEs response was investigated for several hours and the results illustrated that the electrode exhibits a stable response without any chemical or mechanical treatment. The longest test (20 h) involved replicate measurements of the response for a CAP concentration of $5.0 \times 10^{-6} \mathrm{~mol} / \mathrm{L}$ on a dual UMEs in a continuous flow of $0.05 \mathrm{~mol} / \mathrm{L}$ phosphoric acid. The scan rate of $70 \mathrm{~V} / \mathrm{s}$, the accumulation potential of $-300 \mathrm{mV}$, and a accumulation time of $500 \mathrm{~ms}$ were applied for all experiments (Figure 8). The relative standard deviation of the results was $1.8 \%$.

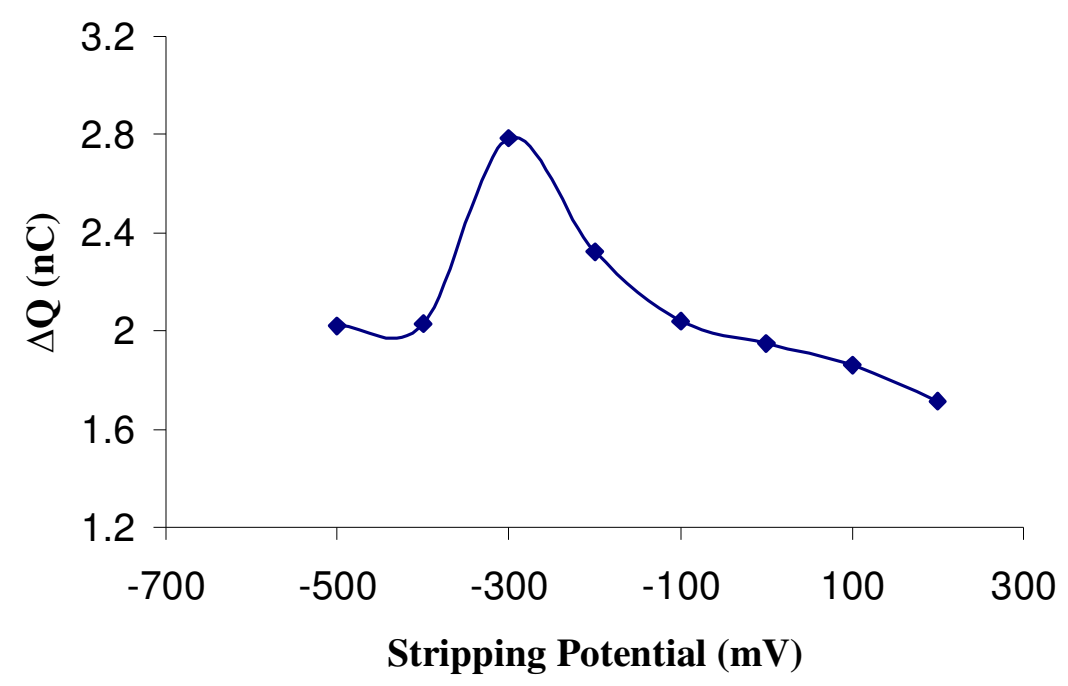

Figure 8. Flow rate effect on the Pt-Au electrode response to the injection of $5.0 \times 10^{-6} \mathrm{~mol} / \mathrm{L}$ Chloramphenicol in $0.05 \mathrm{~mol} / \mathrm{L} \mathrm{H}_{3} \mathrm{PO}_{4}$

\subsection{Precision}

The final mean concentrations were found to be $4.6 \times 10^{-8}, 8.5 \times 10^{-7}$ and $5.3 \times 10^{-6} \mathrm{~mol} / \mathrm{L}$ with associated \%R.S.D. values of 3.4, 2.0 and 0.21 , respectively. The inter-day precision was assessed by injecting the same three concentrations for 3 consecutive days, resulting in mean CAP concentrations $4.4 \times 10^{-8}, 8.7 \times 10^{-7}$ and $5.4 \times 10^{-6} \mathrm{~mol} / \mathrm{L}$ with associated \%R.S.D. values of $3.54,3.22$ and $2.25 \%$, respectively.

\subsection{Calibration curve and detection limit}

Here the experimental conditions were set at the optimum values to obtain the lowest detection limits. Figure 9 presents the calibration graph for the CAP monitoring in $0.05 \mathrm{~mol} / \mathrm{L}$ phosphoric acid. It is significant that in the calculation method the analyte response is proportional to the electrode coverage, similar to the classical stripping voltammetric method. Considering that, may be less obvious when the oxide formation inhibition by adsorbents is considered., For instance, it has been observed that in the presence of species adsorption, a charge decrease in the oxide formation region on the Pt-Au UMEs, is proportional to the surface coverage [44]. Nevertheless, if the rate of mass 
transport controls the electrode coverage, a linear calibration curve is expected up to the point when full electrode coverage is reached during the stripping time. For a high CAP adsorbance, the linear portion of the calibration curve extends from about $8.0 \times 10^{-9}$ to $2.0 \times 10^{-5} \mathrm{~mol} / \mathrm{L}$. Measurements carried out for small analyte concentrations allow the estimation of the detection limit $\mathrm{C}_{\mathrm{DL}}$,

$$
C_{D L}=\frac{3 s_{b}}{m}
$$

where $s_{b}$ is the standard deviation (or noise) of the baseline around the flow injection peak and $\mathrm{m}$ is the sensitivity of the method (a change in the peak height divided by the change in concentration) near the detection limit. finally, the LOQ was obtained to be $6.6 \times 10^{-9} \mathrm{~mol} / \mathrm{L}$. with a resultant \%R.S.D. value of $0.3 \%(n=5)$. In all cases, the accumulation time was $500 \mathrm{~ms}$, but the integration limits in the calculation of the $\Delta \mathrm{Q}$ function were optimized to obtain the maximum $\mathrm{S} / \mathrm{N}$ ratio. The obtained detection limit for CAP was $2.0 \times 10^{-9} \mathrm{~mol} / \mathrm{L}$.

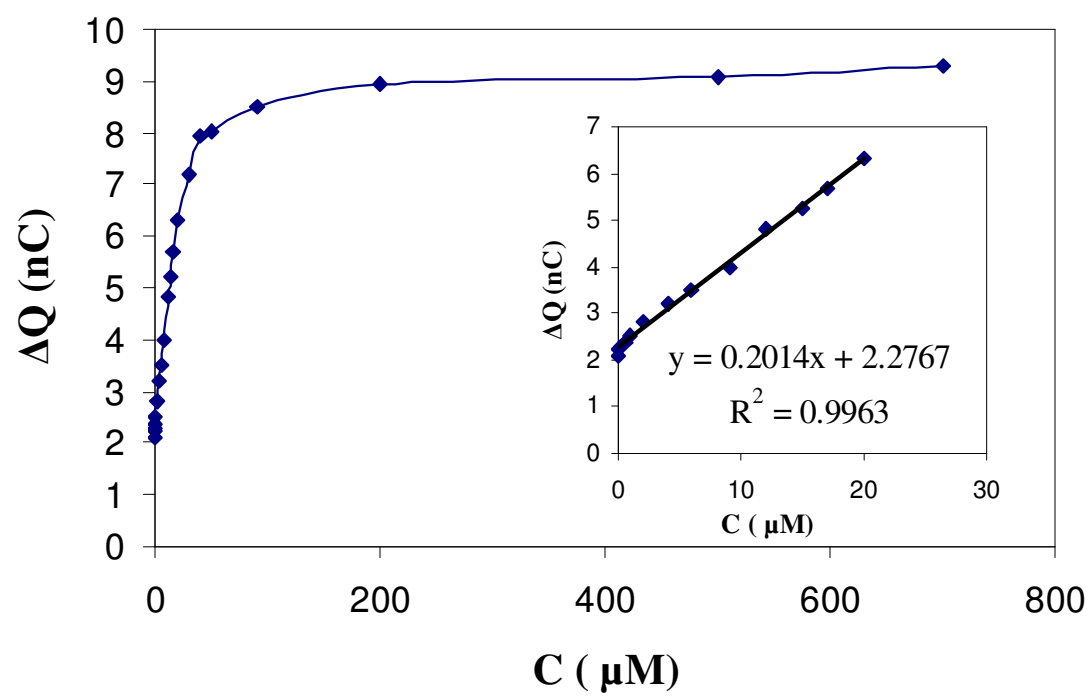

Figure 9. Obtained calibration curves for Chloramphenicol on the Pt-Au electrode in $0.05 \mathrm{~mol} / \mathrm{L}$ $\mathrm{H}_{3} \mathrm{PO}_{4}$

\subsection{Determination of Chloramphenicol in real samples}

The proposed method was also applied to the determination of CAP in spiked urine samples. The results of analysis of spiked urine $(n=5)$ is shown in Table 1 . The results were satisfactory, accurate and precise. No interference was noticed from the urine content, exactly after the dilution with the supporting electrolyte. As can be seen from Table 1, the recoveries are in the range of 98.0 $105.0 \%$. The major advantage of the method as applied to urine is that no prior extraction step is required. 
Table 1. Determination of Chloramphenicol in spiked human urine

\begin{tabular}{|c|c|c|c|c|}
\hline Sample & Added $\left(\mu \mathrm{gL}^{-1}\right) \pm$ S.D. $\%^{\mathrm{a}}$ & Found $\left(\mu \mathrm{gL}^{-1} \pm\right.$ S.D. $\left.\%^{\mathrm{a}}\right)$ & Recovery (\%) & R.S.D $\pm \%(n=5)$ \\
\hline \multirow{4}{*}{ Urine } & $2 \pm 1.0$ & $2.1 \pm 1.4$ & 105.0 & 2.1 \\
\hline & $3 \pm 0.8$ & $3.1 \pm 1.1$ & 103.3 & 1.7 \\
\hline & $5 \pm 1.3$ & $4.9 \pm 1.0$ & 98.0 & 2.1 \\
\hline & $10 \pm 1.0$ & $9.8 \pm 1.5$ & 98.0 & 1.4 \\
\hline
\end{tabular}

${ }^{\mathrm{a}}$ Mean of five measurements

\section{CONCLUSIONS}

1. In this study, it was demonstrated that the CAP concentration in a flowing solution could be measured indirectly via monitoring the current changes during the oxidation and reduction of the electrode surface. More specifically, the S/N ratio was enhanced by applying fast Fourier transformation to the analyte and, then, by signal integration.

2. For the improvement of the sensitivity, the method took advantage of the analyte adsorption on the Pt-Au microelectrode. The influence of the possible adsorbed impurities in the eluent was removed by background subtraction.

3. Therefore, FFTCV can be considered as a new sensitive, accurate and fast method for the determination of similar drugs with adsorption ability on the platinum-gold surface in chromatographic systems, such as HPLC and capillary electrophoresis.

4. However, in order to obtain a better sensitivity for a specific drug, the experimental parameters should be optimized. In addition, such a detection limit (of nanomolar level) made the method suitable for bio-analysis. The major advantage of the designed method, as applied to the urine samples, was that no prior extraction step was required.

\section{ACKNOWLEDGEMENTS}

The authors are grateful to the Research Council of University of Tehran for the financial support of this work.

\section{References}

1. E. H. Allen, J. Assoc. Off. Anal. Chem., 68 (1985) 990.

2. European Regulation 94/1430/CEE.

3. C. van de Water, N. Haagsma, P. J. S. van Kooten and W. van Eden, Z. Lebensm.-Unters.-Forsch., 185 (1987) 202.

4. B. G. Taylor, M. Palomeque, J. V. G. Mateo and J. M. Calatayud, J. Pharm. Biomed., 41 (2006) 347.

5. J. Tuerk, M. Reinders, D. Dreyer, T. K. Kiffmeyer, K. G. Schmidt and H. M. Kuss, J. Chromatogr. B, 831 (2006) 72.

6. A. Wang, L. Zhang and Y. Fang, Anal. Chim. Acta, 394 (1999) 309. 
7. V. Dumonta, A. C. Huet, I. Traynor, C. Elliott and P. Delahaut, Anal. Chim. Acta, 567 (2006) 179.

8. L. Agui, A. Guzman, P. Yanez-Sedeno and J. M. Pingarron, Anal. Chim. Acta, 461 (2002) 65.

9. J. C. Chen, J. L. Shih, C. H. Liu, M. Y. Kuo and J. M. Zen, Anal. Chem., 78 (2006) 3752.

10. L. Kristoffersen, A. Bugge, E. Lundanes and L. Slørdal, J. Chromatogr. B, 734 (1999) 229.

11. P. T. Kissinger and W. R. Heineman, Laboratory Techniques in Electroanalytical Chemistry, Marcel Dekker, New York (1984).

12. M. Paeschke, F. Dietrich, A. Ulig and R. Hintsche, Electroanalysis, 8 (1996) 891.

13. T. Dimitrakopoulos, P. W. Alexander, D. B. Hibbert, Electroanalysis, 8 (1996) 438.

14. R. Hintsche, M. Paeschke, U. Wollenberger, U. Schnakenberg, B. Wagner and T. Lisec, Biosens. Bioelectron., 9 (1994) 697.

15. G. Sreenivas, S. S. Ang, I. Fritsch, W. D. Brown, G. A. Gerhardt and D. Woodward, J. Anal. Chem., 68 (1996) 1858.

16. V. Cosofret, M. Erdosy, T. A. Johnson, R. P. Buck, R. B. Ash and M. R. Neuman, Anal. Chem., 67 (1995) 1647.

17. F. Xiao, F. Zhao, J. Li, R. Yan, J. Yu and B. Zeng, Anal. Chim. Acta, 596 (2007) 79.

18. J. Ermer, J. Pharm. Biomed. Anal., 24 (2001) 755.

19. G. A. Shabir, J. Chromatogr. A, 987 (2003) 57.

20. United States Pharmacopeia 28-NF 23, United States Pharmacopeial Convention Inc., Rockville, MD (2005) 2748.

21. J. C. Miller and J. N. Miller, Statistics for Analytical Chemistry, Ellis Horwood, Chichester, 22 (1984) 82.

22. Z. Al-Kurdi, T. Al-Jallad, A. Badwanamd and A. M. Y. Jaber, Talanta, 50 (1999) 1089.

23. Proceedings of the Internatioal Conference on Harmonisation (ICH), Topic Q2 B: Validation of Analytical Procedures: Methodology, The European Agency for the Evaluation of Medicinal Products, Geneva (1996).

24. P. Norouzi, M. R. Ganjali and P. Matloobi, Electrochem. Commun., 7 (2005) 33.

25. P. Norouzi, G. R. Nabi Bidhendi, M. R. Ganjali, A. Sepehri and M. Ghorbani, Microchim. Acta, 152 (2005) 123.

26. M. R. Ganjali, P. Norouzi, M. Ghorbani and A. Sepehri, Talanta, 66 (2005) 1225.

27. P. Norouzi, M. R. Ganjali, M. Zare and A. Mohammadi, J. Pharm. Sci., 96 (2007) 2009.

28. M.R. Ganjali, P. Norouzi, R. Dinarvand, R. Farrokhi, and A.A. Moosavi-movahedi, Mater. Sci. Eng. C, 28 (2008) 1311.

29. R. M. Wightman and D. O. Wipf, in: A. J. Bard (Ed.), Electroanalytical Chemistry, Marcel Dekker, New York, 15 (1989).

30. J. Lipkowski and L. Stolberg, Adsorption of Molecules at Metal Electrodes, VCH, New York, 1992.

31. P. Norouzi, B. Larijani, M. Ezoddin and M. R. Ganjali, Mater. Sci. Eng. C, 28 (2008) 87.

32. P. Norouzi, M. R. Ganjali, P. Daneshgar, R. Dinarvand, A. A. Moosavi-Movahedi, and A. A Saboury, Anal. Chim. Acta, 590 (2007) 74

33. P. Norouzi, M. R. Ganjali, P. Daneshgar, T. Alizadeh and A. Mohammadi, Anal. Biochem., 360 (2007) 175

34. P. Norouzi, M. R. Ganjali and P. Matloobi, Electrochem. Commun., 7 (2005) 333.

35. M. R. Ganjali, A. Sepehri, A. Daftari, P. Norouzi, H. Pirelahi and A. Moradzadegan, Microchim. Acta, 149 (2005) 245.

36. M. R. Ganjali, P. Norouzi, M. Ghorbani and A. Sepehri, Talanta, 66 (2005) 1225.

37. P. Norouzi, M. R. Ganjali, M. Ghorbani and A. Sepehri, Sens. Actuators B, 110 (2005) 239.

38. P. Norouzi, M. R. Ganjali, S. Shirvani-Arani and A. Mohammadi, J. Pharm. Sci., 95 (2006) 1.

39. M. R. Pourjavid, P. Norouzi, M. R. Ganjali, A. Nemati, H. A. Zamani, M. Javaheri, Int. J. Electrochem. Sci., 4 (2009) 1650.

40. P. Norouzi, M. R. Ganjali and A. A. Moosavi-Movahedi, J. Brazil. Chem. Soc., 18 (2007) 231. 
41. P. Norouzi, M. Qomi, A. Nemati, and M. R. Ganjali, Int. J. Electrochem Sci., 4 (2009) 1248.

42. P. Norouzi, M. R. Ganjali and L. Hajiaghababaei, Anal. Lett., 39 (2006) 1941.

43. P. Norouzi, M. R. Ganjali, S. J. Shahtaheri, R. Dinarvand and A. Hamzehpoor, Chinese J. Chem., 4 (2009) 732.

44. H. Angerstein-Kozlowska, B. E. Conway, B. Barnett and J. Mozota, J. Electroanal. Chem., 75 (1977) 45.

(C) 2010 by ESG (www.electrochemsci.org) 